\title{
mTORC2 is a tyrosine kinase
}

Cell Research (2016) 26:1-2. doi:10.1038/cr.2015.134; published online 20 November 2015

The mammalian target of rapamycin (mTOR), a protein kinase, is the centre of huge attention due to its importance in intracellular signaling and in health and disease. In their recent study, Yin et al. show that mTOR can regulate signaling through the insulin-like growth factor 1 receptor and that it possesses a new enzymatic activity — the ability to phosphorylate proteins on tyrosine residues.

mTOR is a large, multi-domain protein; its catalytic domain resembles that of lipid kinases such as phosphoinositide 3-kinase (PI 3-kinase), but mTOR actually has protein kinase activity, adding phosphate groups to serine or threonine residues in a growing catalog of substrates, many of which are involved in anabolic pathways.

mTOR binds to several protein partners in the cell to form two distinct types of complexes, termed mTOR complexes 1 and 2 (mTORC1/2 [1]). These differ in their protein components, substrate specificity and regulation. For example, mTORC1 is activated by amino acids, and by hormones and growth factors. mTORC1 contains a protein termed Raptor which allows it to phosphorylate substrates such as the ribosomal protein S6 kinases (S6Ks), and this effect is blocked by rapamycin.

mTORC2 contains Rictor in place of Raptor and therefore phosphorylates a distinct set of substrates. These include regulatory (so-called 'hydrophobic') sites in a family of protein kinases which include Akt, also called protein kinase B (PKB). Rapamycin does not directly inhibit mTORC2 function, but can impair it after longer-term treatment [2]. The regulation of $\mathrm{mTORC} 2$ activity remains poorly understood.

mTOR complexes play multifaceted roles in insulin signaling. For example, Akt plays key roles in insulin signaling, mediating the regulation of various proteins involved in the effects of this hormone on metabolism, e.g., glucose transport. Akt signaling indirectly activates mTORC1. In turn, mTORC1 regulates key anabolic processes including protein, lipid and ribosome synthesis. However, mTORC1 can, via the S6Ks, inhibit insulin signaling. This involves the phosphorylation of insulin receptor substrates 1 or 2 (IRS1/2), a crucial link between insulin (and related) receptors and downstream signalling protein, e.g., Akt.

The receptors for insulin (InsR) and insulin-like growth factor I (IGF-IR) are ligand-activated tyrosine kinases, which undergo autophosphorylation allowing them to phosphorylate additional proteins such as IRS1. In turn, phosphorylated IRS1 binds PI 3-kinase; this leads to enhanced production of phosphatidylinositol 3,4,5-trisphosphate, $\mathrm{PIP}_{3}$, and to activation of Akt.

Yin et al. [3] found that rapamycin led to increased phosphorylation of InsR and IGF-IR at key autophosphorylation sites, reflecting increased kinase activity of these receptors.

Knockdown of mTOR or Rictor, or treatment of cells with an inhibitor of mTOR kinase activity, Torin 2, decreased the rapamycin-induced phosphorylation of InsR or IGF-IR, while Raptor knockdown had the converse effect. This indicates the effect requires mTORC2; indeed, the authors show that mTORC2 binds to these receptors, apparently via IRS1/2. However,
mTORC2 does not appear to directly phosphorylate IRS1/2. One possible way in which $\mathrm{mTORC} 2$ increases tyrosine phosphorylation of InsR or IGF-IR is by stimulating the kinase activity of the receptors which then catalyse the phosphorylation of the receptors on tyrosine. The authors ruled this out, by using kinase-dead versions of the receptors or mTOR. Therefore, mTORC2 promotes the tyrosine phosphorylation of InsR/IGF-1R, which is required for downstream signaling from these receptors. While these authors clearly show that rapamycin causes increased phosphorylation of the mTORC2 substrate AKT, earlier studies showed that, at similar time points of treatment in the same cell-type, rapamycin inhibited AKT phosphorylation indicating interference with mTORC2 function [2]. It is not clear how rapamycin promotes mTORC2 function under the conditions used in this study. Another study [4] found that mTORC2 promotes degradation of IRS1, suggesting, in contrast to the conclusions of Yin et al., that mTORC2 can promote insulin resistance. These and other data suggest that the web of interactions between these signaling components is indeed very complex (Figure 1).

mTOR has previously only been reported to act on serine or threonine residues; the present report shows that mTOR can efficiently phosphorylate tyrosines in vitro using either recombinant InsR or peptides as substrate. These data reveal that mTORC2 function is a 'dual-specificity' protein kinase phosphorylating tyrosine as well as serine/ threonine sites. Interestingly, mTORC1 was unable to phosphorylate tyrosines. 


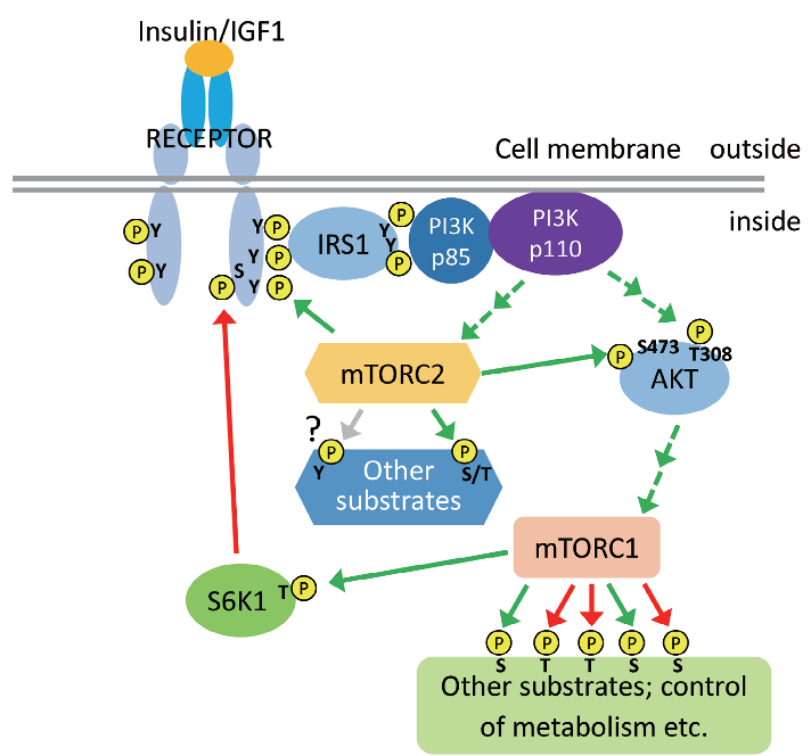

Figure 1 Summary of the signalling connections discussed here, including the new link described by Yin et al. [3] between mTORC2 and the insulin/IGF-1 receptors. Phosphorylation sites are shown schematically (not all are indicated) as ' $P$ ' in a yellow background; $\mathrm{Y}, \mathrm{S}$ and $\mathrm{T}$ indicate tyrosine, serine and threonine respectively. Green and red arrows show activating and inhibitory phosphorylation events respectively. The gray arrow and '?' indicate potential further tyrosine phosphorylation events catalysed by mTORC2. Solid arrows show direct phosphorylation events; dashed lines are indirect signalling links.

Does the mTORC2-stimulated phosphorylation of the InsR/IGF-1R play a role in the actions of the ligands for these receptors? To test this, the authors examined the Rictor knockdown on HepG2 cell proliferation. While this had no effect in the absence of insulin or IGF-1, depletion of Rictor did inhibit proliferation in IGF-1- or insulin-stimulated conditions. Rictor overexpression increased proliferation, an effect that requires the activity of the InsR/IGF-1R.

What are the main implications of these data? First, rapamycin may actually promote signaling from the InsR/ IGF-1R through mTORC2 (as well as via Grb10, a target for mTORC1 itself $[5,6])$ both by the mechanism delineated here and by abrogating the feedback loop from mTORC1 via the S6Ks to IRS1. Second, combining Ins/ IGF-1R receptor inhibitors with $\mathrm{mTOR}$ inhibitors may be a more effective anti-cancer treatment than inhibiting the individual pathways. Third, mTORC2 may phosphorylate additional, so far unidentified proteins on tyrosine, adding to the growing repertoire of $\mathrm{mTOR}$ substrates.

\section{Xuemin Wang ${ }^{1}$, Christopher G Proud ${ }^{1}$}

${ }^{1}$ Nutrition and Metabolism Theme, South Australian Health \& Medical Research Institute, North Terrace, and University of Adelaide, Adelaide, Australia

Correspondence: Christopher G Proud

E-mail: Christopher.Proud@sahmri.com

\section{References}

1 Laplante M, Sabatini DM. Cell 2012; 149:274-293

2 Sarbassov dD, Ali SM, Sengupta S, et al. Mol Cell 2006; 22:159-168.

3 Yin Y, Hua H, Li M, et al. Cell Res 2016; 1:46-65.

4 Kim SJ, DeStefano MA, Oh WJ, et al. Mol Cell 2012; 48:875-887.

5 Yu Y, Yoon SO, Poulogiannis G, et al. Science 2011; 332:1322-1326.

6 Hsu PP, Kang SA, Rameseder J, et al. Science 2011; 332:1317-1322. 